\title{
A Case for Asymmetric-Cell Cache Memories
}

\author{
Andreas Moshovos, Babak Falsafi*, Farid Najm, Navid Azizi \\ Electrical \& Computer Engineering Department \\ University of Toronto \\ * Computer Architecture Laboratory (CALCM) \\ Carnegie Mellon University
}

\begin{abstract}
In this paper, we make the case for building high-performance Asymmetric-Cell Caches (ACCs) that employ recentlyproposed asymmetric SRAMs to reduce leakage proportionally to the number of resident zero bits. Because ACCs target memory value content (independent of cell activity and access patterns), they complement prior proposals for reducing cache leakage that target memory access characteristics. Through detailed simulation and leakage estimation using a commercial $0.13 \mu$ CMOS process model, we show that: (1) on average $75 \%$ of resident data cache bits and $64 \%$ of resident instruction cache bits are zero; (2) while prior research carefully evaluated the fraction of accessed zero bytes, we show that a high fraction of accessed zero bytes is neither a necessary nor a sufficient condition for a high fraction of resident zero bits; (3) the zero-bit program behavior persists even when we restrict our attention to live data, thereby complementing prior leakage-saving techniques that target inactive cells; (4) ACCs can reduce leakage on the average to $23 \%$ compared to a conventional data cache without any performance loss, and to $11 \%$ at the cost of a $5 \%$ increase in overall cache access latency.
\end{abstract}

\section{Introduction}

In this work, we study methods that exploit the memory bitvalue behavior of programs to drastically reduce leakage or static power dissipation in caches without sacrificing performance. Leakage power, or simply leakage, is already a significant fraction of overall power dissipation and is expected to grow by a factor of five every chip generation [3]. It is estimated that in a $0.10 \mu$ technology, leakage power will account for about $50 \%$ of all on-chip power [9]. This increase in leakage is an unwanted side-effect of technological trends: successive processor generations have used more transistors clocked at higher frequencies to improve performance. Keeping the resulting increase in dynamic (or switching) power within limits is possible by scaling down the supply voltage. But, to maintain high operating frequency it is also necessary to scale the transistor threshold voltage $\left(\mathrm{V}_{\mathrm{t}}\right)$, giving rise to subthreshold leakage current and hence leakage power dissipation when the transistor is off [3].

Because leakage is proportional to the number of on-chip transistors and caches account for the dominant fraction of transistors in high-performance designs, in this paper we focus on leakage-aware cache design. There are a number of recent proposals for reducing leakage in caches. Circuitlevel only techniques typically trade off performance for reduced leakage power where this is acceptable - e.g., L2 caches [7]. Proposals to reduce leakage in highperformance caches (e.g., L1 caches in high-performance processors) often use combined circuit- and architecturallevel methods to reduce leakage. These techniques exploit the spatial (how much data) and temporal (for how long) characteristics of the memory reference stream of typical programs to reduce leakage in those parts of the cache that are left unused for long periods of time $[6,8,10,15,16]$.

In this paper, we look beyond the time and space characteristics of program memory behavior. We offer a new degree of freedom in reducing leakage by exploiting the memory value content at the bit level. We evaluate Asymmetric-Cell Caches (ACCs) that drastically reduce leakage even when most of the cache is actively used. At the core of ACCs is a set of asymmetric SRAMs that are designed to drastically reduce leakage when they store a zero (bit) while maintaining high performance. A circuitlevel analysis of a family of asymmetric SRAMs was presented recently $[1,2]$. While asymmetric SRAMs provide the means to reduce leakage in any generalized SRAM-based structure (e.g., cache memories, register files, TLBs, etc.), it is the memory value behavior of programs that determines the actual potential for leakage reduction in cache memories using ACCs. In this paper we study the cache-resident memory value behavior of ordinary programs and show the leakage benefits possible in various applications. This work complements the previous studies that looked only at the circuit-level aspects of ACCs.

A number of studies have shown that the data processed and the instructions fetched by processors contain many zeros, e.g., [4,5,14]. These studies have focused on the dynamic memory stream (i.e., the values read or written by the processor) and at larger than a bit granularities (e.g., bytes). But all cache cells contribute to leakage equally independently of how often they are accessed by the processor. In this paper, we show that a high dynamic frequency of zero bytes is neither a necessary nor a sufficient condition for reducing leakage using ACCs, and the potential for ACCs is greater than that indicated by the fraction of zero bytes in the dynamic memory stream.

The rest of this paper is organized as follows. In section 2 we comment on related work. In section 3 we discuss the rationale behind ACCs, explain how they relate to existing leakage reduction methods and present our analysis of memory value behavior. In section 4 , we review the asymmetric SRAM cell family that forms the core of the ACCs and present leakage reduction results. Finally, in section 5 we summarize our findings.

\section{Related Work}

Numerous architectural techniques for reducing power in high-performance caches have been proposed. We restrict 
our attention to those proposals that target leakage. Many recent proposals to reduce leakage in caches employ a supplygating circuit-level mechanism called gated- $V_{\mathrm{dd}}[15]$ to effectively "turn off" cache cells that remain inactive for long execution periods. Yang et al., proposed the DRI cache [12] which dynamically resizes and turns off portions of a cache. Kaxiras et al., proposed Cache Decay [10] which turns off individual blocks using expiration counters. Zhou et al., extend cache decay by dynamically extracting the expiration count value during runtime [16]. With all aforementioned methods, care must be taken to avoid disabling blocks that are live since doing so will increase miss rate and potentially dynamic power dissipation (misses will be serviced by the much larger higher levels of the memory hierarchy). Flautner et al., propose Drowsy Caches [6] a variation on cache decay that obviates the need to turn off and evict cache blocks by putting potential dead blocks into "sleep" using supply-voltage-scaling. Heo et al., propose floating the bitlines in unused subarrays of a sequentially-precharged cache to reduce bitline leakage [8]. As we show in section 3.4, our technique is orthogonal to all aforementioned methods. Even if it was possible to perfectly predict which blocks are not live, ACCs can reduce leakage significantly. Moreover, even when most cache blocks hold live data ACCs can reduce leakage power significantly.

To the best of our knowledge, no previous proposals on using bit values to reduce leakage power dissipation exists. Villa et al., present dynamic zero compression [13] where zero values are exploited to reduce dynamic power dissipation. ACCs attack leakage dissipation and rely on bit values. As we show in section 3.3, even if zero bytes were completely disabled, our method could still reduce leakage significantly. Moreover, we show that the behavior exploited by the two techniques is fundamentally different. Several circuit-level only proposals for reducing leakage power in caches exist. These exploit multiple or dynamic threshold voltages and/or supply voltages and transistor stacking. In their majority, these techniques trade-off performance for reduced power dissipation and are oblivious to application behavior.

\section{Exploiting Data Values to Reduce Leakage}

Ideally, cache cells would be as fast as possible consuming as little leakage power as possible. Unfortunately, this requirement is increasingly at odds with a fundamental technology trade off: as the supply voltage gets smaller, fast transistors tend to dissipate high leakage power. One of the key circuit-level mechanisms for reducing leakage is to use "weaker" transistors (e.g., having higher threshold voltage $\left(\mathrm{V}_{t}\right)$ or higher length to width ratio). Unfortunately, "weaker" transistors are also slower and the resulting performance loss is unacceptable for high-performance caches.

Asymmetric-Cell Caches (ACCs) exploit memory value content to reduce leakage in high-performance caches. ACCs are built using a family of novel SRAM cells (proposed in $[1,2]$ ) built on the following premise: weaken (asymmetrically) only those transistors necessary to drastically reduce leakage when the cell stores a zero while maintaining high performance. Compared to conventional, high-performance SRAM cells, the asymmetric cells dissipate lower leakage in both states. However, the reduction in leakage is much higher when they store a "zero" as opposed to when they store a "one". In section 4, we review the SRAM cell family and extend the previous analysis by evaluating four ACCs under realistic program workloads. A detailed, circuit-level analysis of asymmetric SRAM appears in [1,2] and is beyond the scope of this paper.
What makes ACCs successful is the fraction of resident zero bits in typical program behavior. In the rest of this section, we carefully analyze the bit-value program behavior and show that most (but not all) of the programs we studied exhibit a strong bias towards zero bits.

\subsection{Methodology}

We use Simplescalar v3.0 to simulate a state-of-the-art superscalar processor with a two-level cache hierarchy. Table 1 depicts our base processor configuration. We assume split level-one data (L1D), level-one instruction (L1I) caches and a unified level-two (L2) cache. We present results for systems with an L1I and an L1D of 32Kbytes each (32-byte blocks, 2way set-associative) and an L2 of 1 Mbytes (64-byte blocks, 4way set-associative). We only present results on bit-value characterization in the data arrays of level-one caches. We have also experimented with characterizing bit values in L2 and found the L2 results quite similar to L1D results due to: (a) SPEC2000's tiny instruction footprints allowing the data streams to dominate occupancy in L2, and (b) SimpleScalar v3.0 only simulating a uniprogrammed environment with a single application's footprint in L2. Similarly, we do not present a bit-value characterization for the cache tag arrays despite finding the values to be highly skewed. This is because SimpleScalar v3.0 does not simulate address translation and hence tag values are artificially skewed towards zero. We used the following SPEC CPU2000 benchmarks: gzip, swim, applu, vpr, gcc, mesa, art, mcf, equake, ammp, parser, gap, vortex, bzip, and twolf. We also included mpeg2encode from mediabench. The binaries were compiled for the Alpha 21264 architecture using Compaq's compilers and for the Digital Unix V4.0F. We simulated two billion committed instructions or to completion (whichever happened first) after skipping the initialization. To account for variations in bit-value distributions across compilations and instruction sets, we also present results for binaries compiled for PISA, the MIPS-like instruction set simulated by Simplescalar v3.0. We used gcc version 2.7.2 and we also ported GNU's g77 FORTRAN compiler to produce PISA binaries for the FORTRAN benchmarks we studied. In all experiments we consider only cache lines that have been touched at least once by the program.

Table 1. Processor configuration.

\begin{tabular}{|c|c|}
\hline Branch Predictor & Fetch Unit \\
\hline $\begin{array}{c}\text { 16k GShare+16K bi-modal } \\
\text { with 16K selector }\end{array}$ & $\begin{array}{c}\text { Up to } 8 \text { instr. per cycle. } \\
\text { 64-entry Fetch Buffer } \\
10 \text { cycles misprediction penalty. }\end{array}$ \\
\hline Scheduler & Issue/Decode/Commit \\
\hline $\begin{array}{c}\text { 128 insts and 64 loads/stores } \\
\text { Perfect Disambiguation }\end{array}$ & $\begin{array}{c}\text { Any instructions / cycle } \\
4 \text { loads/stores per cycle }\end{array}$ \\
\hline L1/UL2 Access Latencies & Main Memory \\
\hline $3 / 16$ cycles & Infinite, 100 cycles \\
\hline
\end{tabular}

\subsection{Bit-Value Distribution}

Figure 1(a) illustrates the bit-value distribution in L1 caches including a breakdown in terms of the address space these bits belong to. On average, almost $75 \%$ and $64 \%$ of bit values are zero in the data and instruction caches respectively. Not surprisingly, the instruction cache does not exhibit much variation in the distribution of zeros across applications. Furthermore, while zero is the common bit value, there is not a strong bias. Unlike instruction caches, data caches exhibit a large variation in the skew towards zero. The breakdown of bits across the data, heap, and stack segments indicate that except for three applications, gzip, applu, and swim, the 
majority of zero-bit distributions are from the heap. Gzip maintains its main data structures statically in the data segment. Applu and swim are FORTRAN77 applications and therefore do not use the heap. The rest of the applications dynamically allocate their data structures.

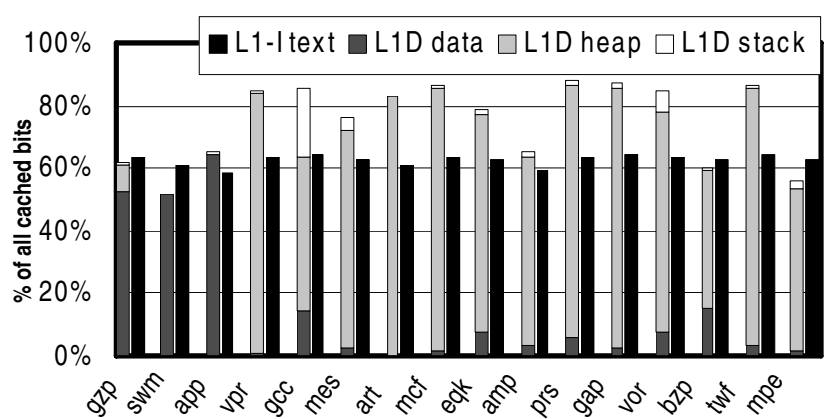

(a) $32 \mathrm{~K}$

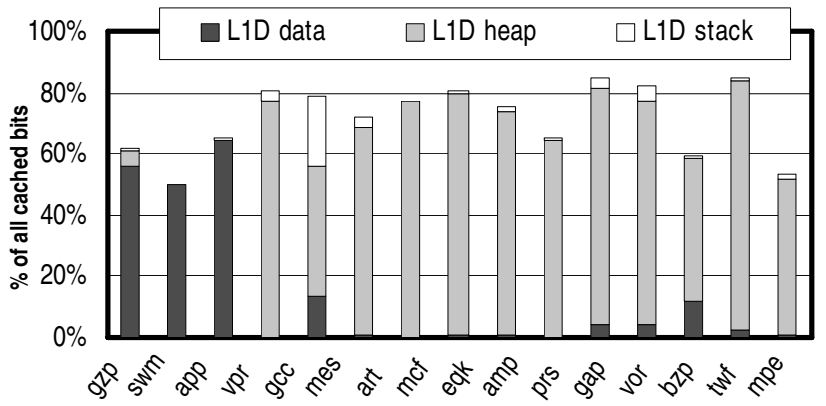

(b) 32K PISA

Figure 1: (a) Fraction of cache bits that are zeroes for $L 1 D$ and L1I for the ALPHA binaries for 32-Kbyte caches. (b) Same for

PISA binaries and for 32-Kbyte L1D caches. These PISA experiments do not include the benchmark gap, because the PISA capable gcc compiler cannot produce code for this benchmark.

The data cache results can be divided into three groups: (1) the applications with predominantly dynamic data structures and a significant skew towards a high fraction of zero bits, (2) the compression applications, and (3) the dense numerical (floating-point) applications. Vpr, gcc, mesa, art, mcf, equake, parser, gap, vortex, and twolf all heavily use dynamic memory allocation. Many of the heap objects are heavily biased towards zero and are actively used. Heap objects often contain small positive integer values and pointers. Also compilers often align aggregates of structures to prevent misaligned accesses, padding aggregates with fields that remain zero. The second group of applications, bzip, gzip and mpeg2encode, compress the input streams and as such reduce the longevity of large sequences of zero bits in the data cache during execution. The third group of applications, amp, applu, and swim are dense numerical computations, in which bit values are more uniformly distributed across zeros and ones. In the extreme case, swim bit values are almost equally distributed across zeros and ones. While we do not show these results we note that using $64 \mathrm{~K} \mathrm{~L} 1$ caches resulted in very similar distribution of zero bits.

The next experiment was set up to identify how sensitive the cache bit-value behavior of programs is to the specific instruction set architecture. Figure 1 (c) shows the bit-value distributions for L1D caches for PISA binaries. We do not compare the bit-value distributions in the instruction cache because PISA uses a 64-bit instruction format that is highly skewed towards zero. The results indicate a slight increase in the skew towards zero when using the Alpha binaries. Unlike PISA, Alpha uses 64-bit integer and floating-point values.

\subsection{Dynamic vs. Cache Resident Bit-Value Behavior}

Prior work carefully evaluates the dynamic occurrence of zeros in the memory access stream [11,14] and the datapath [4,5]. Our key observation is that unlike prior work, ACCs target: (1) the behavior of cache resident (rather than accessed) values, and (2) a high fraction of zero bits even if an application's cache-resident data exhibit a small fraction of zero bytes. In this section, we make the case that a high dynamic occurrence of zeros is neither necessary nor sufficient for ensuring a large fraction of zero bits in the cache.

Figures 2(a) and 2(b) show a breakdown of the cache data byte values in terms of the number of their bits that are "one" (range 0 to 8). Part (a) shows this breakdown for all cache resident data values while part (b) does the same for the dynamic data reference stream. To understand the cache resident metric consider that in the trivial example of a cache with one line with the value all ones for one cycle and all zeroes for nine cycles, the cache-resident percentage of zero-bits is $90 \%$. The dynamic frequency of zero bytes is not correlated to the fraction of zero bytes in the data cache. In some programs the fraction of zero bytes seen by the processor is much larger than that of the data stored in the cache. In other programs, the reverse is true. For example in twolf, $41 \%$ of the bytes accessed is zero, while $61 \%$ of the bytes in the cache are zero. The relatively frequency of static vs. dynamic zero bytes is reversed for gzip where $40 \%$ of the bytes accessed vs. only $20 \%$ of the cache bytes are zeros.

There are applications such as swim, bzip and mpeg2encode that have very few zero bytes in the data cache and that access very few zero bytes also. Still, even in these applications a large fraction of cache bits are zero suggesting that there is great potential for ACCs. For example, in swim more than half of the cache bits are zero while only $4 \%$ of the bytes are zero. Figure 2 parts (c) and (d) illustrate the same byte-level distribution for instruction caches. The graphs indicate that zero distributions across bytes are more uniform in instruction caches than data caches limiting the opportunity for zero-byte compression.

Figure 3 shows a breakdown of the zero bits in the various memory segments for the data cache. Two bars are shown per program. The left bar is for the cache resident values while the right is for the dynamic memory reference stream. At first it may seem that the dynamic fraction of zeros follows closely the fraction of zero bits in the cache. However, these measurements show that the relative importance of various data values is different across the two streams. This can be clearly seen for stack data. In virtually all programs, stack data have a negligible impact on the fraction of zero bits in the data cache. In the dynamic stream, however, stack values are responsible for many of the zero bits.

\subsection{Bit Values in Live Data}

Recent architectural/circuit cache leakage reduction techniques $[10,15]$ have used supply-gating [12] or supply-voltage-scaling [6] to reduce leakage in the inactive or "dead" cache lines. Asymmetric cells can be used in conjunction with these techniques to reduce leakage in both the "live" and "dead" cache lines. Figure 4 illustrates the fraction of zero bits in L1D and L1I in the live cache lines - i.e., lines that will be accessed again prior to being evicted. The graphs indicate that, 


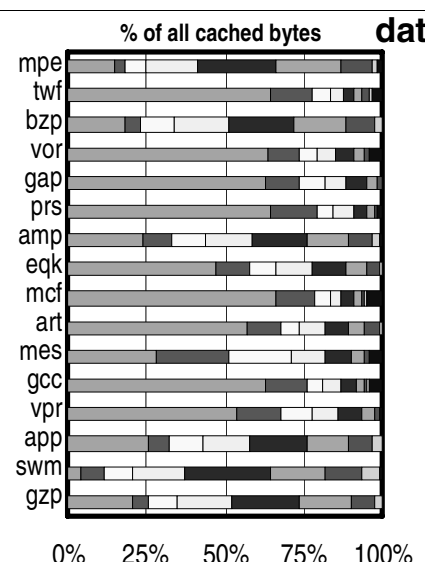

(a) Cache-Resident

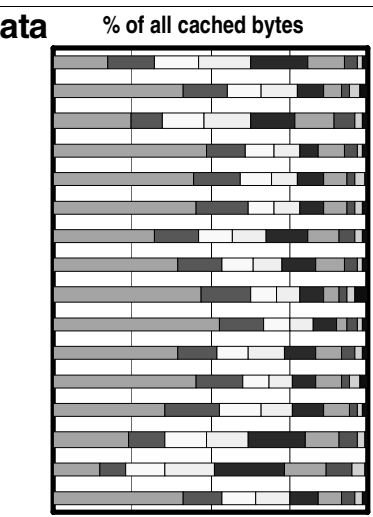

$25 \% \quad 50 \% \quad 75 \% \quad 100 \%$

(b) Dynamic

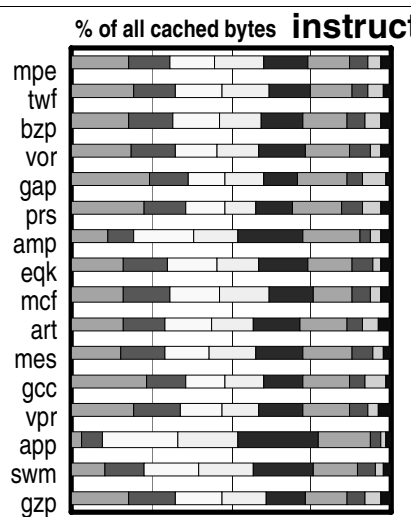

$\begin{array}{lllll}0 \% & 25 \% & 50 \% \quad 75 \% \quad 100 \% & 0 \%\end{array}$

(c) Cache-Resident

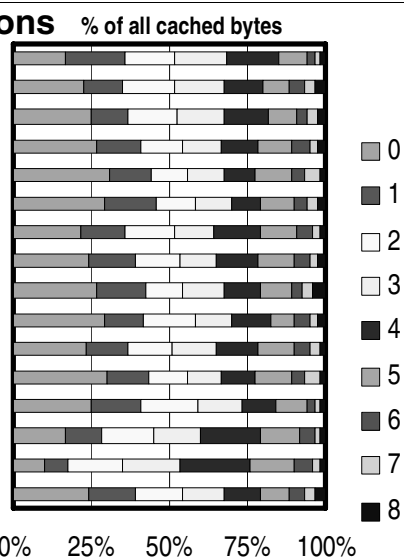

(d) Dynamic

Figure 2: Per-byte bit distribution. The figures show the breakdown of bytes with respect to the number of their bits that are one (range is 0 to 8). (a) Data cache resident bytes. (b) Data cache bytes as read by the processor. (c) Instruction cache resident bytes. (d) Instruction cache bytes as accessed by the processor.

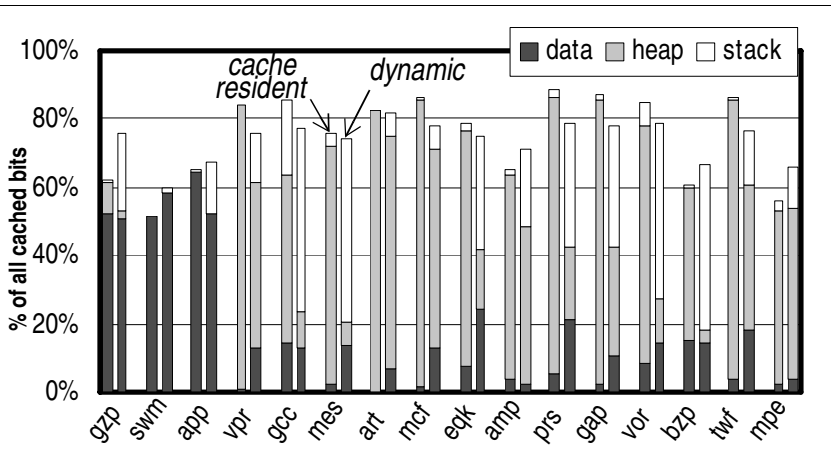

Figure 3: Which memory segment zero bits come from for ALPHA binaries: left bar is for the cache resident values; right bar is for the dynamic memory reference stream.

as expected, there is little change in the distribution of zeros in the instruction stream.

\section{Asymmetric SRAM}

The key enabling mechanism for value-based leakage optimization is a set of asymmetric SRAM cells. Here we review their principle of operation. The interested reader can find a detailed, circuit-level analysis of the complete asymmetric SRAM family in [1,2]. Let us first review where leakage is dissipated in the conventional high-performance six regular- $\mathrm{V}_{\mathrm{t}}$ transistor cell shown in Figure 5(a). The cell comprises two inverters (MP2, MN2) and (MP1,MN1) and two pass transistors MN3 and MN4. In the inactive state, the wordline (WL) is held at " 0 " so that the two pass transistors are off isolating the cell from the two bitlines BL and BLb. At this stage the bitlines are also typically charged at $\mathrm{V}_{\mathrm{dd}}$ (e.g., logical one). The cells spend most of their time in the inactive state. In this state, most of the leakage is dissipated by the transistors that are off and that have a voltage differential across their drain and source. Which are those transistors depends on the value stored in the cell. When the cell is storing a zero (left side) the leaking transistors are MP2, MN1 and MN3 (figure 5(a)). Figure 5(b) shows an asymmetric cell where the MP2, MN1 and MN3 have been replaced with high$\mathrm{V}_{\mathrm{t}}$ transistors. The leakage of this cell is comparable to the high- $\mathrm{V}_{\mathrm{t}}$ cell in the preferred state and comparable to the regular- $\mathrm{V}_{\mathrm{t}}$ cell otherwise.

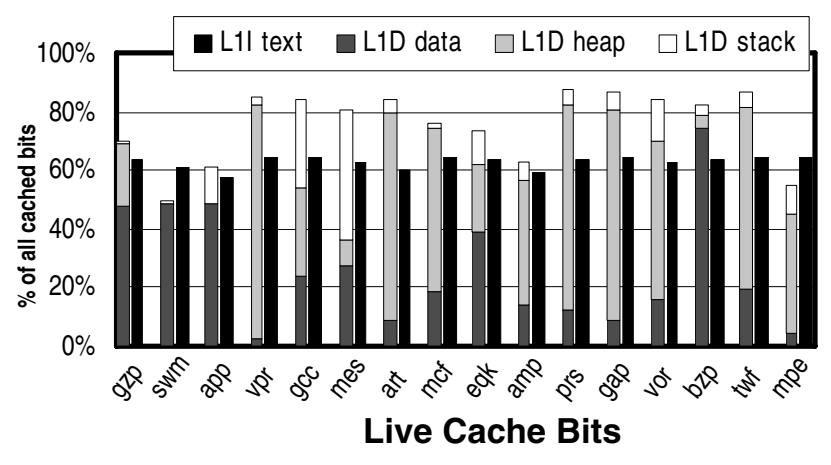

Figure 4: Fraction of caches bits that are zeroes for L1D and L1I blocks that are "live" and for the ALPHA binaries

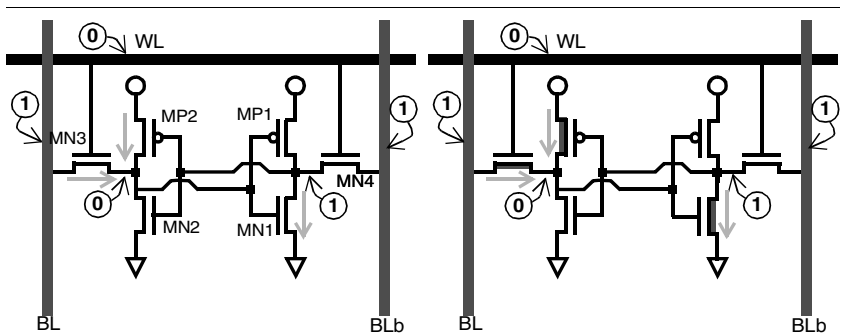

(a) Regular-Vt Cell

(b) Basic Asymmetric

Figure 5: (a) A conventional regular- $V_{t} S R A M$ cell. (b) An asymmetric, dual- $V_{t}$ cell.

The basic asymmetric cell of figure 5 (b) serves to illustrate the idea behind asymmetric cells but suffers in terms of stability and access latency. A family of asymmetric cells with various performance, leakage and stability characteristics is presented in [2]. The characteristics of the better cells are summarized in table 2 normalized over the Regular-Vt cell (first row). We consider four cells: (i) Leakage-Enhanced (LE), (ii) SpeedEnhanced (SE), (iii) Stability-Leakage Enhanced (SLE), and (vi) Stability-Speed Enhanced (SSE). Compared to the basic 
asymmetric cell, these cells offer different performance vs. leakage reduction ratios. We report leakage reduction in both states $(20 \%$ means a reduction of $5 \mathrm{X})$, increase in latency and two metrics of stability: signal to noise margin (SNM) and the current necessary to trip the cell's value. In the last two characteristics, a positive percentage suggests an increase in stability while a negative percentage a decrease. There is no clear best asymmetric cell if we consider all characteristics. For example, stability-wise SSE is best, however, leakage-wise LE is far better. None of these cells introduces an area overhead. We should note that SE and LE exhibit a relative asymmetry in their stability characteristics. While they improve one stability metric over the conventional cell, they exhibit reduced stability in the other metric. Overall, the stability differences are minor and all cells are usable under worst-case assumptions and for the commercial $0.13 \mu \mathrm{m}$ process that we used.

Table 2. Characteristics of Asymmetric Cells.

\begin{tabular}{|c|c|c|c|c|c|}
\hline & Leakage (0) & Leakage (1) & $\Delta$ Delay & $\Delta$ SNM & $\Delta \mathbf{I}_{\text {trip }} / \mathbf{I}_{\text {read }}$ \\
\hline Regular-Vt & $100 \%$ & $100 \%$ & $0 \%$ & $0 \%$ & $0 \%$ \\
\hline LE & $1 \%$ & $14 \%$ & $5 \%$ & $7 \%$ & $-5 \%$ \\
\hline SE & $14 \%$ & $50 \%$ & $0 \%$ & $-6 \%$ & $15 \%$ \\
\hline SLE & $14 \%$ & $43 \%$ & $5 \%$ & $23 \%$ & $-7 \%$ \\
\hline SSE & $50 \%$ & $53 \%$ & $0 \%$ & $9 \%$ & $13 \%$ \\
\hline
\end{tabular}

\subsection{Leakage Power Reduction}

Figure 6 shows the leakage power dissipation for the four ACCs. Due to space limitations we report results only for the data cache. We report leakage power as a fraction of the leakage power dissipation of the regular $-V_{t}$ cell cache. This metric includes only the power dissipated by the data array cells. This is appropriate given that Simplescalar does not model a multiprogrammed environment and as a result tag bits are strongly skewed towards zero. We also measured leakage in the decoder and found that it represents a very small fraction of overall cache leakage power. Furthermore, the leakage dissipated by the decoder array is the same for the conventional cache and the ACCs. As expected, on the average, the LE cell offers the highest average savings ( $11 \%$ or about $10 \mathrm{X}$ ) but these come at the expense of a $5 \%$ increase in cache latency. SE and SLE perform similarly $(22.9 \%$ and $21.2 \%$ respectively). Finally, the SSE that has the best stability characteristics offers only a $2 \mathrm{X}$ reduction in leakage.

\section{Conclusion}

Contrary to existing architectural techniques for reducing cache leakage power that focus on the time and space characteristics of the memory reference stream we targeted its value content. The key enabling technology for our work is a set of asymmetric SRAM cells that dissipate drastically reduced leakage when they store a "zero". This work complements the recent work on the circuit-level design of ACCs $[1,2]$ by demonstrating that the memory value behavior of programs is such that asymmetric cell caches can effectively reduce leakage power without impacting performance. Moreover, we demonstrated ACC's utility for a broad spectrum of applications and even if it would be possible to accurately identify those cache parts that are left unused. One

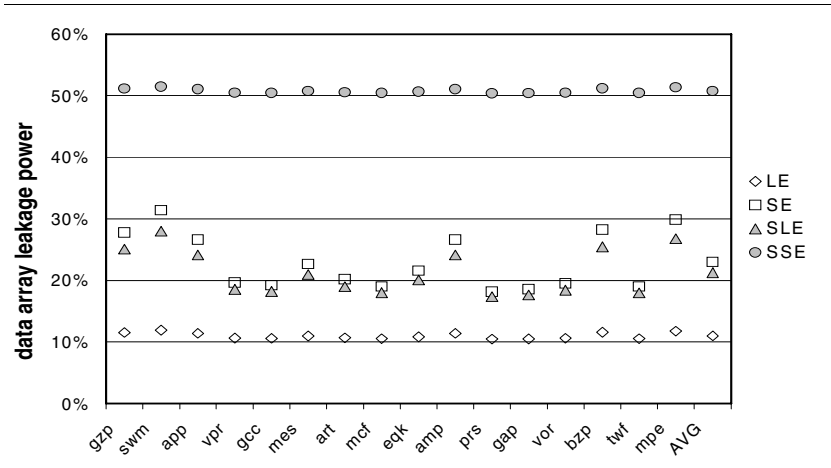

Figure 6: Data cache leakage power with the asymmetric cells relative to the regular- $V_{t}$ conventional cache. Lower is better.

of the major insights of this work is that the behavior exploited by ACCs is not the same as the one exploited by recent zero value related work for reducing dynamic power in various parts of processors.

\section{References}

[1] N. Azizi, A. Moshovos, and F. Najm. Low-leakage asymmetric SRAM. Proceedings of the 2002 International Symposium on Low Power Electronics and Design, Aug. 2002.

[2] N. Azizi, F. Najm and A. Moshovos,. Low-leakage asymmetric SRAM. IEEE Transactions on VLSI Systems, Aug. 2003.

[3] S. Borkar. Design challenges of technology scaling. IEEE Micro, 19(4):23-29, July 1999.

[4] D. Brooks and M. Martonosi. Dynamically exploiting narrow width operands to improve processor power and performance. In Proceedings of the Fifth IEEE Symposium on High-Performance Computer Architecture, pages 13-22, Jan. 1999 .

[5] R. Canal, A. Gonzalez, and J. E. Smith. Very low power pipelines using significance compression. In Proceedings of the 33rd Annual IEEE/ACM Int'l Symposium on Microarchitecture, Dec. 2000

[6] K. Flautner, N. S. Kim, S. Martin, D. Blaauw, and T. Mudge. Drowsy caches: Simple techniques for reducing leakage power. In Proceedings of the 29th Annual International Symposium on Computer Architecture, May 2002.

[7] F. Hamzaoglu, Y. Ye, A. Keshavarzi, K. Zhang, S. Narendra, S. Borkar, M. Stan, and V. De. Dual-Vt SRAM cells with full-swing single-ended bit line sensing for high-performance on-chip cache in $0.13 \mathrm{um}$ technology generation. In Proceedings of the 2000 International Symposium on Low Power Electronics and Design (ISLPED), pages 15-19, July 2000.

[8] S. Heo, K. Barr, M. Hampton, and K. Asanovic. Dynamic fine-grain leakage reduction using leakage-biased bitlines. In Proceedings of the 29th Annual Intt'l Symposium on Computer Architecture, May 2002.

[9] T. Kam, S. Rawat, D. Kirkpatrick, R. Roy, G. S. Spirakis, N. Sherwani, and C. Peterson. EDA challenges facing future microprocessor design. IEEE Transactions on Computer-Aided Design, 19(12), Dec. 2000

[10] S. Kaxiras, Z. Hu, and M. Martonosi. Cache decay: Exploiting generational behavior to reduce cache leakage power. In Proceedings of the 27th Annual International Symposium on Computer Architecture, July 2000.

[11] M. Lipasti. Value Prediction and Speculative Execution. $\mathrm{PhD}$ thesis, Carnegie Mellon University, Apr. 1997.

[12] M. D. Powell, S.-H. Yang, B. Falsafi, K. Roy, and T. N. Vijaykumar. GatedVdd: A circuit technique to reduce leakage in cache memories. In Proceedings of the 2000 International Symposium on Low Power Electronics and Design (ISLPED), pages 90-95, July 2000.

[13] G. Reinman and N. Jouppi. An integrated cache timing and power model. Technical report, Compaq Corporation, Western Research Laboratory, 1999.

[14] L. Villa, M. Zhang, and K. Asanovic. Dynamic zero compression for cache energy reduction. In Proceedings of the 33rd Annual IEEE/ACM Int'l Symposium on Microarchitecture, Dec. 2000.

[15] S.-H. Yang, M. D. Powell, B. Falsafi, K. Roy, and T. N. Vijaykumar. An integrated circuit/architecture approach to reducing leakage in deep-submicron high-performance i-caches. In Proceedings of the Seventh IEEE Symposium on High-Performance Computer Architecture, Jan. 2001.

[16] H. Zhou, M. C. Toburen, E. Rotenberg, and T. M. Conte. Adaptive mode control: A static-power-efficient cache design. In Proceedings of the Ninth International Conference on Parallel Architectures and Compilation Techniques, 2001. 